www.jmscr.igmpublication.org

Impact Factor (SJIF): 6.379

Index Copernicus Value: 71.58

ISSN (e)-2347-176x ISSN (p) 2455-0450

crossref DOI: https://dx.doi.org/10.18535/jmscr/v6i6.102

Journal Of Medical Science And Clinical Research

IGM Publication

An official Publication of IGM Publication

\title{
Comparison of Percutaneous Nephrolithotomy (PCNL) and Retrograde Intrarenal Surgery (RIRS) for Management of Inferior Calyceal 1-2 cm Stones: A Matched Pair Analysis
}

\author{
Authors \\ Dr Gyan Prakash Singh ${ }^{1}$, Dr Pradeepta Kumar Panda ${ }^{2}$ \\ ${ }^{1}$ Asso. Prof., ${ }^{2}$ Mch resident \\ Department of Urology, S.C.B. Medical College \& Hospital , Cuttack, Odisha.India \\ Correspondng Author \\ Dr Pradeepta Kumar Panda \\ Email: drkitusraban@gmail.com, Mobile no-9437031992
}

\begin{abstract}
Objective: To compare percutaneous nephrolithotomy (PCNL) and retrograde intrarenal surgery (RIRS) for the treatment of lower calyceal calculi with diameter of $1-2$.

Method: 59 patients who had renal stones ranging from 2 to $4 \mathrm{~cm}$ in diameter were treated with RIRS. The outcomes of these patients were compared with patients who underwent PCNL using matched-pair analysis (1:1 scenario). The matching parameters were the size, number and location of the stones as well as age, gender, body mass index, solitary kidney, degree of hydronephrosis, presence of previous shock wave lithotripsy and open surgery.

Results: The two study groups were comparable for demographic characteristics and stone characteristics. It was found that RIRS is comparable to PCNL as a treatment modality in inferior calyceal renal stones of size $1-2 \mathrm{~cm}$. The efficacy as defined by stone clearance rate was similar in the two procedures. RIRS had a shorter hospital stay, lesser morbidity and complications compared to PCNL.

Conclusion: RIRS is an effective and safe alternative to PCNL in the treatment of inferior calyceal stones of size $1-2 \mathrm{~cm}$. The choice of surgical technique between PCNL and RIRS should depend upon the expertise and choice of the surgeon as well as take the financial capability of the patient into account.
\end{abstract}

\section{Introduction}

Renal stone disease is one of the most common urological diseases of modern society. With westernisation of global culture, however the site of stone formation has migrated from lower to upper urinary tracts ${ }^{1}$. According to the National Health and Nutrition Survey 2012, 10.6\% of men and $7.1 \%$ of women in USA are affected by renal stone disease, compared to just $6.3 \%$ of men and
$4.1 \%$ women that were affected in $1994 .^{2}$ Further, within the affected population the gender gap has narrowed substantially. Historically stone disease affected adult men more than adult women. However recent data suggest that this difference of incidence is narrowing. As regards to race, among U.S. men highest prevalence was found in whites followed by Hispanics, Asians and African-Americans. The geographical distribution 
of stone disease tends to roughly follow environmental factors. ${ }^{3}$

Treatment modality of renal stones depends upon stone size, location, symptoms and any other anatomical variation in calyceal system of kidney. Treatment can be medical in form of dietary management, disease specific therapies, and medical expulsion therapy (MET) of stones $^{3}$. Surgical treatment options include Extra Corporeal Shock Wave Lithotripsy (ESWL), Percutaneous nephrolithotomy (PCNL), Retrograde Intra Renal Surgery (RIRS), Laparoscopic stone retrieval and open stone surgery. ${ }^{4,5,6}$

For lower calyceal stones $1-2 \mathrm{~cm}$ in size SWL, RIRS and PCNL are the available treatment modalities $^{7}$. SWL is minimally invasive, without anaesthesia requirement, has a good patient tolerance with adequate analgesia. Though it is an easy treatment option for stones less than $1 \mathrm{~cm}$ in size, its efficacy and stone clearance rate decreases with the increments of stone size ${ }^{8}$. PCNL is regarded as a highly effective procedure with consistently high stone-free rates and its result does not depend on anatomic factors and stone size; gives good stone clearance with minimal complications and lower retreatment rate though it is associated with higher morbidity. Retrograde Intrarenal Surgery (RIRS) is a less invasive and feasible treatment option, and has also short hospitalization time, low morbidity and complication rate. It may be an alternative of PCNL in the treatment Lower Pole calculi with diameter smaller than $2 \mathrm{~cm}^{9}$.

In this study, we compared the outcomes of RIRS, and PCNL for 1-2 cm lower calyceal calculi, using a prospective randomized design.

\section{Material \& Methods}

This prospective randomised interventional study was conducted at SCB medical college and Hospital, Cuttack, Odisha from November 2015 to November 2017.

Patients of stone diseases attending Urology OPD, SCB medical college and Hospital, between November 2015 to November 2017 with single inferior calyceal stone of size 1-2 $\mathrm{cm}$ and who satisfy the eligibility criteria (inclusion and exclusion criteria). The study was started after getting ethical approval from the institutional ethics committee of SCB medical college and Hospital. Written informed consent was taken from all the patients before enrolling them for the study. Patients between 15-75 years of age with single inferior calyceal stones of size $1-2 \mathrm{~cm}$ were included in study. Patients with bleeding disorders, active urinary infection (untreated), Age $>75$ years and < 15 years, weight $>100 \mathrm{Kg}$ and $<$ $30 \mathrm{Kg}$, comorbid cardiovascular and respiratory illnesses were excluded from study. The patients were categorized into 2 groups:

Group 1: Retrogradeintrarenal surgery (RIRS) using Karl-Storz 7.5 french flexible ureterorenoscope (FLEX-X2)

Group 2: PCNL using 26 french KarlStorznephroscope.

Computer generated randomisation table was used to allocate the patients who fulfilled inclusion criteria and were not excluded to RIRS or PCNL arm of the study. In the current study we have taken the sample size as 30 in PCNL group and 29 in RIRS group by computer generated formula for non-inferiority randomised controlled trial. All patients were registered with all relevant data including name, age, sex, height, weight, indoor registration number, occupation, address, phone number, date of admission, date of surgery, and date of discharge. Preoperative evaluation included detailed clinical history including associated medical conditions if any, past medical or surgical intervention if any. Clinical evaluation included detailed general physical examination and systemic examination in detail. Investigations included urine routine $\&$ microscopy examination, culture \& sensitivity, complete haemogram, kidney function test, liver function test, ultrasound of kidney, ureter, bladder (KUB), contrast enhanced computer tomography of KUB and all other investigations required by anaesthetist. Sterile urine was mandatory in both the groups before proceeding for surgery. Peri-operative 
evaluation included operative time, fluoroscopy time, blood transfusion rate indicating amount of haemorrhage and pain score using visual analogue scale. Early postoperative evaluation included hospitalisation days. Requirement of additional treatment sessions for the same disease (renal stone) were evaluated in terms of auxiliary treatment rates and retreatment rates. PCNL in our study groups were done either with conventional technique i.e. placement of both nephrostomy tubes and double $\mathrm{J}$ stents or tubeless technique i.e. double J stent used only. All RIRS patients were stented with double $\mathrm{J}$ stents post operatively. Auxiliary procedure were defined as any procedure required in addition to primary modality to make the patient stone free as in PCNL group, like ureteroscopy to remove migrated ureteral stone and extra corporeal shock wave lithotripsy (ESWL) to remove residual stones. ${ }^{10} \quad$ In case of RIRS auxiliary procedures likely to be needed were stenting after RIRS and subsequent removal of stent, ESWL for residual stone, rigid uretroscopy for ureteral stones. ${ }^{11}$ Retreatment was defined as same modality of treatment needed as to render stone free either early post postoperatively or within 3 month follow up. Stone clearance rates were evaluated both early and in follow-up at 3 months. Early evaluation included intraoperative examination both fluoroscopically and visually using either nephroscope in PCNL group or flexible ureteroscope in RIRS group and X ray KUB in the next postoperative day. A mandatory non-contrast computerised tomography (NCCT) KUB was done in follow up after 3 months in all study group patients to look for residual or recurrent stones. Post-operative complications were evaluated in terms of severe pain, haemorrhage, urinary tract infection, bowel or ureter injury and any other complication related to surgery were noted. All patients were shifted toward after keeping them under observation of anaesthetist for few hours. In PCNL group nephrostomy tubes were removed after urine becomes clear followed by catheter removal .In RIRS group catheters were removed in postoperative day 1 . Double J stent removal done in all patients after 3 week. All patients were discharged as early as possible when they were pain free and declared fit to go home. All patients were followed up after one week post procedure and after 3 months. After 3 months all patients were investigated with NCCT scan to document stone free rate (SFR) at 3 months.

\section{Statistical Analysis}

Data was collected in the pre-determined format. All the data was coded and entered into a master spreadsheet on MS Office Excel 2010 (Microsoft Corporation, Seattle, USA. 2010). Proforma were checked for correctness and completeness before entering the data. The data was later transferred from MS Excel to SPSS (IBM SPSS Statistics 21.0; IBM SPSS, 2012) for analysis. The mean/ median and standard deviations were calculated for continuous variables such as age, height, weight and other continuous variables and proportions (percentage) were calculated for discrete variables. The unpaired t-test was used to detect the difference between continuous variables in the two intervention arms. Pearson's chi-square test and Fisher's exact test were used to calculate significance of differences between proportions in case of discrete variables. A p-value $<0.05$ was considered as significance.

\section{Results}

The two study groups had a similar distribution of patients with regards to demographic characteristics of age, sex, weight and height. (table-1) 
Table 1: Distribution of demographic characteristics of study participants

\begin{tabular}{|l|c|c|c|}
\hline Demographic parameter & $\begin{array}{c}\text { RIRS Group } \\
(\mathbf{n = 2 9})\end{array}$ & $\begin{array}{c}\text { PCNL Group } \\
(\mathbf{n}=\mathbf{3 0})\end{array}$ & p value \\
\hline Mean age in years $( \pm$ SD) & $45.17 \pm 11.53$ & $47.87 \pm 11.32$ & $0.36^{*}$ \\
\hline Sex $($ Male $)$ & $18(62.08 \%)$ & $21(70 \%)$ & $0.519^{\wedge}$ \\
\hline Mean weight in $\mathrm{Kg}( \pm \mathrm{SD})$ & $63.24 \pm 10.39$ & $61.53 \pm 11.45$ & $0.55^{*}$ \\
\hline Mean height in cm $( \pm$ SD) & $164.36 \pm 10.39$ & $166.47 \pm 7.34$ & $0.29^{*}$ \\
\hline
\end{tabular}

*Unpaired $t$-test

$\wedge$ Pearson's chi squared test

Table 2: Pre-operative stone characteristics in the two study groups

\begin{tabular}{|l|c|c|c|}
\hline Stone characteristics & $\begin{array}{c}\text { RIRS Group } \\
(\mathbf{n = 2 9})\end{array}$ & $\begin{array}{c}\text { PCNL Group } \\
(\mathbf{n}=\mathbf{3 0})\end{array}$ & \multirow{2}{*}{ p value } \\
\hline $\begin{array}{l}\text { Stone side: Left } \\
\text { Number }(\%) \text { Right }\end{array}$ & $12(41.29 \%)$ & $16(53.33 \%)$ & \multirow{2}{*}{$0.358^{*}$} \\
\cline { 2 - 3 } Mean stone diameter in mm $( \pm$ SD) & $17(58.62 \%)$ & $14(46.67 \%)$ & \multirow{2}{*}{$0.44^{\wedge}$} \\
\hline & $15.06 \pm 3.7$ & $15.8 \pm 3.5$ & \\
*Pearson's chi squared test \\
$\wedge$ Unpaired t-test
\end{tabular}

In the RIRS group, 12(41.29\%) patients have left sided stone and $17(58.62 \%)$ patients have right sided stone. In the PCNL group, renal stone was left sided in 16(53.33\%) patients and right sided in14 $(46.67 \%)$ patients. This difference in the location of stone according to side was not statistically significant (Pearson's chi-squared test; $\mathrm{p}=0.358$ ).

In the RIRS group the mean stone diameter was $15.06 \mathrm{~mm}( \pm 3.7 \mathrm{~mm})$ and in the PCNL group mean stone diameter was $15.8 \mathrm{~mm}( \pm 3.5 \mathrm{~mm})$. This difference in mean stone size among the two study groups was not statistically significant (Unpaired t-test; $\mathrm{p}=0.44)$. (table 2$)$

Table 3: Peri-operative data of the two study groups

\begin{tabular}{|c|c|c|c|}
\hline Peri-operative parameter & $\begin{array}{c}\text { RIRS Group } \\
(\mathbf{n}=29)\end{array}$ & $\begin{array}{c}\text { PCNL Group } \\
(\mathbf{n}=\mathbf{3 0})\end{array}$ & p value \\
\hline Mean operative time in minutes $( \pm$ SD) & $70.59 \pm 10.14$ & $78.17 \pm 12.89$ & $0.07^{\wedge}$ \\
\hline Mean fluoroscopy time in seconds $( \pm$ SD) & $64.13 \pm 9.07$ & $157.17 \pm 15.46$ & $<0.001^{\wedge}$ \\
\hline Transfusion requirement & 0 & $6(20 \%)$ & $0.02 *$ \\
\hline Mean Visual analogue score $( \pm$ SD $)$ & $1.58 \pm 0.57$ & $3.6 \pm 1.38$ & $<0.001^{\wedge}$ \\
\hline
\end{tabular}

In the RIRS group the mean fluoroscopy time was $64.13 \mathrm{sec}( \pm 9.07 \mathrm{sec})$ while it was higher in the PCNL group with a mean duration of $157.17 \mathrm{sec}$ $( \pm 15.46 \mathrm{sec})$. This difference in mean fluoroscopy time among the two study arms was statistically significant (Unpaired t-test; $\mathrm{p}<0.001$ ). Intraoperative blood transfusion was required in 6 patients $(6.7 \%)$ overall. All of these 6 patients were in the PCNL group. In the RIRS group no patients required blood transfusion while in the PCNL group 6 patients (20\%) needed intra- operative blood transfusion. This difference in proportions between the two study arms was statistically significant (Fisher's exact test; $\mathrm{p}=$ 0.02). The mean pain score in terms of visual analogue score in the RIRS group was $1.58 \pm 0.57$ while it was higher in the PCNL group, with a mean score $3.6 \pm 1.38$. This difference in mean pain scores between the two study arms was statistically significant (Unpaired t-test; $\mathrm{p}<$ 0.001). (table-3)

Table 4: Hospital stay duration of patients among the two study groups

\begin{tabular}{|l|c|c|c|}
\hline Parameter & $\begin{array}{c}\text { RIRS Group } \\
(\mathbf{n = 2 9})\end{array}$ & $\begin{array}{c}\text { PCNL Group } \\
(\mathbf{n = 3 0})\end{array}$ & p value \\
\hline Mean hospital stay duration in days (SD) & $2.86 \pm 0.99$ & $4.37 \pm 2.11$ & $<0.001 *$ \\
\hline Unpaired $t$-test
\end{tabular}


The mean duration of hospital stay was 2.86 days ( \pm 0.99 days $)$ in the RIRS group and4.37 days $( \pm 2.11$ days) in the PCNL group. This difference in mean hospital stay was statistically significant (Unpaired t-test; $\mathrm{p}<0.001$ ). (table-4)

Table 5: Requirement of additional surgical procedures in the study groups

\begin{tabular}{|l|c|c|c|}
\hline Parameter & $\begin{array}{c}\text { RIRS Group } \\
(\mathbf{n = 2 9 )}\end{array}$ & $\begin{array}{c}\text { PCNL Group } \\
(\mathbf{n = 3 0 )}\end{array}$ & p value \\
\hline Auxiliary procedure(s) required & $1(3.4 \%)$ & $3(10 \%)$ & $0.612 *$ \\
\hline Retreatment required within 3 months & $2(6.8 \%)$ & $1(3.33 \%)$ & $0.612 *$ \\
\hline * Fisher's exact test
\end{tabular}

Auxiliary procedures was needed in $1(3.4 \%)$ of the patients in RIRS group and $3(10 \%)$ patients in PCNL group, but there is no statistical difference (Fisher's exact test; $p=0.612$ ). ESWL was used as auxiliary procedure in all the 4 patients. Retreatment within 3 months after surgery was required in $3(5.1 \%)$ patients out of total 59 patients. In the RIRS group, 2 patients (6.8\%) needed retreatment while in the PCNL group only 1 patient $(3.3 \%)$ needed retreatment. This difference in proportions of those needing retreatment was not statistically significant (Fisher's exact test; $p=0.612$ ). (table 5)

Table 6: Stone clearance observed in the study groups

\begin{tabular}{|l|c|c|c|}
\hline Parameter & $\begin{array}{c}\text { RIRS Group } \\
(\mathbf{n = 2 9 )}\end{array}$ & $\begin{array}{c}\text { PCNL Group } \\
(\mathbf{n = 3 0 )}\end{array}$ & p value \\
\hline Stone clearance immediate post-op & $26(89.66 \%)$ & $28(93.3 \%)$ & $0.67 *$ \\
\hline Stone clearance at 3 month post-op & $27(93.2 \%)$ & $29(96.7 \%)$ & $0.61^{*}$ \\
\hline
\end{tabular}

Stone clearance was observed immediately postoperative and on 3 month follow-up. Immediate post-operative stone clearance was seen in 26 (89.66\%) patients in RIRS group and 28(93.3\%) patients in PCNL group but no statistical difference between them (Fisher's exact test; $p$
$=0.67)$. On 3 month follow-up, stone clearance was 27 patients (93.2\%) RIRS group and in 29 patients $(93.3 \%)$ in PCNL group, but statistically no difference between them (Fisher's exact test; $p$ $=0.61)$. (table 6$)$

Table 7: Complications observed in study participants in the two study groups

\begin{tabular}{|c|c|c|c|}
\hline Complication observed & $\begin{array}{c}\text { RIRS Group } \\
(\mathbf{n}=\mathbf{2 9})\end{array}$ & $\begin{array}{c}\text { PCNL Group } \\
(\mathbf{n}=\mathbf{3 0})\end{array}$ & p value \\
\hline prolonged pain & $1(3.4 \%)$ & $12(40 \%)$ & $0.001 *$ \\
\hline Urinary tract infection & $2(6.8 \%)$ & $1(3.3 \%)$ & $1 *$ \\
\hline Haematuria & $1(3.4 \%)$ & $3(10 \%)$ & $0.612 *$ \\
\hline
\end{tabular}

Complications that were seen in the post-operative period were prolonged pain requiring analgesics more than 5 days, urinary tract infection (UTI) and haematuria. (table-7)

\section{Discussion}

The pre-operative stone characteristics in both the groups were also comparable. In the RIRS group, $12(41.29 \%)$ patients have left sided stone and 17 $(58.62 \%)$ patients have right sided stone. In the
PCNL group, renal stone was left sided in 16 $(53.33 \%)$ patients and right sided in14(46.67\%) patients. This difference in the location of stone according to side was not statistically significant (Pearson's chi-squared test; $\mathrm{p}=0.358$ ).

Intra-operative blood transfusion was required in 6 patients $(6.7 \%)$ overall. All of these 6 patients were in the PCNL group. In the RIRS group no patients required blood transfusion while in the PCNL group 6 patients (20\%) needed intra- 
operative blood transfusion. This difference in proportions between the two study arms was statistically significant (Fisher's exact test; $\mathrm{p}=$ 0.02). This is comparable to results of Kursad Zengin et $\mathrm{al}^{12}$ (The mean decrease in haemoglobin level was $1.4 \pm 0.9 \mathrm{~g} / \mathrm{dl}$ in the PNL group and 0.3 $\pm 0.1 \mathrm{~g} / \mathrm{dl}$ in the RIRS group $\square<0.001$ ) and that of $A$ kman et $\mathrm{al}^{13}(2$ patients out of $34,5.88 \%$ needed transfusion in PCNL group and none in RIRS group).

The mean pain score in the RIRS group was $1.58 \pm 0.57$ while it was higher in the PCNL group, with a mean score $3.6 \pm 1.38$. This difference in mean pain scores between the two study arms was statistically significant (Unpaired t-test; $\mathrm{p}<$ 0.001). This finding is again comparable the findings of Sabnis et $\mathrm{al}^{14}$.

On 3 month follow-up,stone clearance was 27 patients $(93.2 \%)$ RIRS group and in 29 patients (93.3\%) in PCNL group, but statistically no difference between them (Fisher's exact test; $p$ $=0.61$ ). This result is comparable to that of Sabnis et $\mathrm{al}^{14}$ (The stone clearance rates in the microperc and RIRS groups were similar (97.1 vs $94.1 \%, \mathrm{P}=1.0$ ). The mean duration of hospital stay was 2.86 days ( \pm 0.99 days) in the RIRS group and 4.37 days ( \pm 2.11 days) in the PCNL group. This difference in mean hospital stay was statistically significant (Unpaired t-test; $\mathrm{p}$ $<0.001)$. This finding is comparable to that of Kursad Zengin et $\mathrm{al}^{12}$ (The mean hospital stay was $2.3 \pm 1.3$ days in the PNL group and $1.1 \pm 0.4$ days in the RIRS group). Amr S. Fayad et al ${ }^{15}$ in their study titled "Tubeless mini-percutaneous nephrolithotomy versus retrograde intrarenal surgery for lower calyceal stones of $2 \mathrm{~cm}$ has found similar findings. Hongyang Jiang $^{16}$ et al 2017in there Meta-analysis showed that miniPCNL group led to a higher SFR [odds ratio: 1.96; 95\% confidence interval: 1.46-2.64;] but brought a larger postoperative decrease in hemoglobin levels compared with RIRS. RIRS provided a shorter hospital time. There was no significant difference in operation time. Higher postoperative complications were detected in the mini-PCNL, but the difference was not significant. Grade I and III complications did not vary between two procedures, but grade II complications were of lower incidence in RIRS group. In the light of these results, compared with RIRS, mini-PCNL provided significantly higher SFR and efficiency quotient for managing calculi; however, it resulted in higher incidence of postoperative complications, larger haemoglobin drops, and longer hospital stay.

\section{Conclusion}

In this study it was found that RIRS is comparable to PCNL as a treatment modality in inferior calyceal renal stones of size $1-2 \mathrm{~cm}$. The efficacy as defined by stone clearance rate was similar in the two procedures. RIRS had a shorter hospital stay, lesser morbidity and complications compared to PCNL. The choice of surgical technique between PCNL and RIRS should depend upon the expertise and choice of the surgeon as well as take the financial capability of the patient into account.

\section{References}

1. Nikolaos Ferakis and Marios Stavropoulos et al. Mini percutaneous nephrolithotomy in the treatment of renal and upper ureteral stones: Lessons learned from a review of the literature. Urol Ann. 2015 Apr-Jun; 7(2): 141-148.

2. Helal M, Black T, Lockhart J, Figueroa TE. The Hickman peel-away sheath: Alternative for paediatric percutaneous nephrolithotomy. J Endourol. 1997; 11:171-2.

3. Jackman S., Docimo S., Cadeddu J., Bishoff J., Kavoussi L., Jarrett T. (1998a) The 'mini-perc' technique: a less invasive alternative to percutaneous nephrolithotomy. World J Urol 16: 371374.

4. Sabnis R., Ganesamoni R., Ganpule A., Mishra S., Vyas J., Jagtap J., et al. (2013) Current role of microperc in the 
management of small renal calculi. Indian J Urol 29: 214-218.

5. Shashikant Mishra, et al .Tubeless mini percutaneous nephrolithotomy in infants and preschool children: a preliminary report. J Urol. 2010.;24:1935-9

6. Janak Desai,Solanki, R. et al"A Novel Technique of Ultra-Mini-Percutaneous Nephrolithotomy: Introduction and an Initial Experience for Treatment of Upper Urinary Calculi Less Than $2 \mathrm{~cm}$," BioMed Research International, vol. 2013, Article ID 490793.

7. Park J,Bumsik Hong, Taehan Park, HyungKeun Park et al, Effectiveness of noncontrast computed tomography in evaluation of residual stones after percutaneous nephrolithotomy. J Endourol. 2007 Jul; 21(7):684-7.

8. Darsan Sharma et al .The "All-Seeing Needle": Initial Results of an Optical Puncture System Confirming Access in Percutaneous Nephrolithotomy. European journal Urology. Jun 2011, Vol. 59, No. 6: 1054-1059.

9. Turk C, Knoll T, Petrik A, et al. Guidelines on Urolithiasis. European Association of Urology,2012.

10. Wing-hang AU. Retrograde Intrarenal Surgery (RIRS)- Ureterorenoscopic Lithotripsy for Renal Stones VOL.14 NO.10 OCTOBER 2009 medical bulletin .

11. Mahajan P M, Padhye A S, et al Is stenting required before retrograde intrarenal surgery with access sheath. Indian $\mathbf{J}$ Urol2009;25:326-8

12. Kursad Zengin, Serhat Tanik, Nihat Karakoyunlu, Nevzat Can Sener, Sebahattin Albayrak, Can Tuygun, Hasan Bakirtas, M. Abdurrahim Imamoglu and Mesut Gurdal. Retrograde Intrarenal Surgery versus Percutaneous Lithotripsy to Treat Renal Stones 2-3 cm in Diameter. Hindawi Publishing Corporation BioMed
Research International Volume 2015, Article ID 914231 .

13. T. Akman, M. Binbay, F. Ozgor et al., "Comparison of percutaneous nephrolithotomy and retrograde flexible nephrolithotripsy for the management of 2$4 \mathrm{~cm}$ stones: a matched-pair analysis," BJU International, vol. 109, no. 9, pp. 1384-1389, 2012.

14. Sabnis R., Ganesamoni R., Ganpule A., Mishra S., Vyas J., Jagtap J., et al. (2013) Current role of microperc in the management of small renal calculi. Indian J Urol 29: 214-218.

15. AmrS.Fayad, Mohamed G.Elsheikh, Waleed Ghoneima Tubeless minipercutaneous nephrolithotomy versus retrograde intrarenal surgery for lower calyceal stones of $\leqslant 2 \mathrm{~cm}$ : A prospective randomised controlled study .Arab J Urol. 2017 Mar; 15(1): 36-41.

16. Hongyang Jiang, Zhe Yu, Liping Chen, Tao Wang, Zhuo Liu, Jihong Liu, Shaogang Wang, and Zhangqun Ye . Minimally Invasive Percutaneous Nephrolithotomy versus Retrograde Intrarenal Surgery for Upper Urinary Stones: A Systematic Review and MetaAnalysis. Bio Med Research International, Volume 2017, Article ID 2035851. 